\title{
AVALIAÇÃO DO ESTADO NUTRICIONAL DE TRÊS GRAMADOS ORNAMENTAIS EM ILHA SOLTEIRA-SP: UM ESTUDO DE CASO
}

EVALUATION OF THE NUTRITIONAL STATUS OF THREE ORNAMENTAL LAWNS IN ILHA SOLTEIRA-SP: A CASE STUDY

\author{
Nathália Batista de Oliveira \\ Engenheira Agrônoma - UNESP Ilha Solteira-SP \\ nahhbatista8@gmail.com \\ João Francisco Veroneze de Oliveira \\ Engenheiro Agrônomo - UNESP Ilha Solteira-SP \\ joao.veronezeoliveira@gmail.com \\ Patrick Luan Ferreira dos Santos \\ Mestrando em Agronomia, Unesp Ilha Solteira-SP \\ patricklfsantos@gmail.com \\ Raíssa Pereira Dinalli Gazola
}

Prof. contratada Doutora, UNESP Ilha Solteira/SP, departamento de Fitotecnia, tecnologia de alimentos e sócio-economia raissadinalli@gmail.com

\section{Regina Maria Monteiro de Castilho}

Prof. Doutora, UNESP Ilha Solteira/SP, departamento de Fitotecnia, tecnologia de alimentos e sócio-economia castilho@agr.feis.unesp.br

\begin{abstract}
RESUMO
Os gramados são de grande importância para o meio ambiente, podendo ser utilizados para diversos fins, contudo, para que apresentem visual agradável é necessário que sejam muito bem cuidados tanto no momento da implantação como depois, na manutenção. Sendo assim, objetivou-se com o presente trabalho avaliar o estado nutricional de gramados ornamentais, analisando, em laboratório, as quantidades de macro e micronutrientes no solo e nas folhas da grama esmeralda (Zoysia japonica Steud.). O estudo foi conduzido na cidade de llha Solteira - SP, no mês de maio de 2016, em três lugares, sendo duas residências e uma instituição de ensino particular. Foram avaliados a necessidade de adubação, bem como o índice de conteúdo de
\end{abstract}


clorofila (ICC) com um clorofilômetro manual e sua relação com os macro e micronutrientes foliares. Com base nos resultados obtidos, conclui-se que os gramados avaliados apresentaram boas condições nutricionais. Nestes, a adubação de manutenção nem sempre é necessária, caso os restos provenientes do corte da grama esmeralda não sejam retirados. O clorofilômetro pôde indicar, de forma mais rápida e prática, a necessidade de fertilização, podendo ser utilizado por empresas de manutenção paisagística para saber a hora certa de adubação.

Palavras-chave: Arquitetura paisagística; Implantação e manutenção de áreas gramadas; Uso de gramados no paisagismo; Grama esmeralda.

\begin{abstract}
The lawns have significant importance for the environment and can be used for various purposes, nevertheless, to have a pleasant look of them it is necessary to be very well kept during both time, i.e. at the of implantation and afterward in maintenance. Therefore, the focus of this work is to evaluate the nutritional status of ornamental lawns by analyzing in a laboratory the macro and micronutrient content in the soil and leaves of Emerald Grass (Zoysia japonica Steud.). The work was performed in Ilha Solteira City, SP, in May 2016, in three places, two residences and one private educational institution. It was evaluated the need for fertilization, as well as the index of chlorophyll content (ICC), using a hand chlorophyllometer, and its relationship with macro and foliar micronutrients. It was concluded, based on the results achieved, that the evaluated lawns presented good nutritional conditions. The maintenance fertilization is not even necessary when Emerald Grass cutting wastes are not removed. The chlorophyllometer indicated, in a faster and practical way, the need for fertilization, and could be used by landscape maintenance companies to know the right time of applying fertilizers.
\end{abstract}

Keywords: Landscape Architecture; Grassy Areas Implantation and Maintenance; Use of Lawns in Landscaping; Emerald Grass. 


\section{INTRODUÇÃO}

Os gramados são de grande importância para o meio ambiente e nesse contexto, podem ser utilizados como revestimento vegetal do solo (contra erosão), removem e armazenam as impurezas do ar, servem como fonte contínua de oxigênio e ar fresco, e são amplamente utilizados na arquitetura paisagística, por serem implantados na composição de áreas verdes como jardins residenciais e comerciais. Além disso, trazem benefícios, como o conforto térmico em relação ao asfalto. (GODOY; VILLAS BÔAS, 2010).

Um gramado bonito e bem manuseado, segundo Brito (2010), traz as pessoas uma atração especial por ele, produz prazer e admiração por quem o visita; já um mal cuidado transmite uma sensação de descuido e abandono.

Gramados ornamentais estão estritamente relacionadas com a arquitetura paisagística, pois segundo Zanon (2003), eles são empregados em jardins residenciais, áreas esportivas, obras públicas, parques industriais, etc. Sendo que no Brasil, para esse tipo de uso, os gramados mais cultivados são Esmeralda ou Japonesa (Zoysia japonica Steud.), a Santo Agostinho ou Inglesa (Stenotaphrum secundatum Kuntze), a Bermuda (Cynodon dactylon L.) e a São Carlos (Axonopus afins Chase).

As espécies acima citadas, de acordo com Gurgel (2003), são de clima quente e as que mais se adaptam às condições climáticas brasileiras, por apresentarem capacidade de se desenvolver em altas temperaturas, apesar de algumas variedades tolerarem geadas esporádicas e outras baixas temperaturas, mas sempre acima de zero graus Celsius.

Assim, em um projeto de paisagismo, é essencial conhecer a espécie de grama a ser implantada, pois segundo Winters (2012) gramados ornamentais são as primeiras plantas a serem lembradas na execução de um projeto, e ainda é notório seu uso em residências recém acabadas e nas inaugurações de obras públicas.

Contudo, para que os gramados desempenhem seu papel, proporcionando uma paisagem mais agradável, um local adequado para a prática de esportes ou protegendo o meio ambiente, é necessário que estes sejam adequadamente implantados.

O plantio da grama é uma das fases mais importantes no estabelecimento de um gramado em um projeto paisagístico, pois, o objetivo é que este permaneça por muitos anos no local em que foi instalado. 
A nutrição e a adubação, para a maioria das culturas, são essenciais, porém, para as gramas, estes dois fatores são mais importantes para as características qualitativas (GODOY; VILLAS BOAS, 2003).

Devido à grande utilização dessas áreas verdes em residências e comércios, percebe-se a necessidade de estudar a qualidade de gramados. Portanto, o objetivo do presente trabalho foi avaliar as condições dos gramados ornamentais na cidade de llha Solteira - SP.

\section{IMPORTÂNCIA DOS GRAMADOS}

Os gramados ornamentais são de grande importância para o meio ambiente, pois liberam oxigênio, reduzem a emissão de gás carbônico, controla a poluição do solo, e ainda, possuem grande valor estético, proporcionando locais confortáveis e seguros para diversão e prática de esportes (GODOY; VILLAS BÔAS, 2010). Além disso, utilizar gramados não se trata somente de valor estético agregado, mas sim da melhoria do conforto térmico; pois a absorção de calor no processo de transpiração e redução da radiação e reflexão dos raios solares diminui a temperatura do ar (PEDRON, 2002).

É preferível que opte-se por utilizar gramado natural, visto que a grama artificial apresenta temperatura superficial muito elevada, podendo atingir $64^{\circ} \mathrm{C}$ em dias muito quentes (OLIVEIRA et al., 2016).

Segundo Zanon (2003), há uma grande variedade de grupos de consumidores de grama no Brasil, podendo ser citados: jardins residenciais, áreas esportivas, obras públicas, parques industriais. No ano de 2010, o Brasil atingiu uma área de 17000 hectares cultivados de grama, sendo que a produção concentrava no estado de São Paulo, seguido pelo Paraná e Minas Gerais; entretanto o cultivo ocorre em quase todos os estados do país. Hoje, o mercado de gramas tem uma área com produção de grama legalizada estimada em 25000 ha, sendo São Paulo e Paraná os principais Estados produtores, com um faturamento estimado de 500 milhões de reais na produção agrícola de grama cultivada (ANDRADE, 2016).

Segundo o Zanon (2015), a região de Itapetininga é a maior produtora de grama do país. No entanto, o lucro caiu de 70 a $80 \%$ para $30 \%$, devido ao aumento dos insumos. 


\section{USO DE GRAMADOS ORNAMENTAIS NA ARQUITETURA PAISAGÍSTICA}

Gramados ornamentais, são as principais espécies de plantas utilizadas em projetos paisagísticos em todo o mundo (WINTERS, 2012). Isso se deve pois eles interagem com os demais elementos, como árvores, arbustos, canteiros, fontes, etc., servindo harmoniosamente como pano de fundo ao cenário (MACIEL et al., 2008). Assim, desempenham um excelente efeito estético aos parques e jardins (GODOY; VILLAS BÔAS, 2010).

Dentre as espécies mais utilizadas na arquitetura paisagística, está a grama esmeralda para áreas de intenso pisoteio, grama São Carlos para locais semi-sombreados e grama Santo Agostinho, utilizado em cidades litorâneas, onde o solo apresenta maior salinidade (WIINTERS, 2012).

Ainda, além de serem implantadas em projetos residenciais, as espécies de gramas, estão sendo amplamente utilizadas nas chamadas calçadas ecológicas (SANTOS et al., 2015). Esse tipo de revestimento do solo, consiste em evitar a impermeabilização dos passeios públicos e privados, através da implantação de material permeável como os concregramas, entretravados e tapetes de gramas. Onde juntamente com uma arborização adequada no calçamento, fará com que a cidade fique valorizada no aspecto estético, quebrando um pouco da frieza das ruas (ANGELIS et al., 2007).

Outra utilização de gramados ornamentais na arquitetura paisagística, é na sua implantação em telhados verdes, posto que de acordo com Santos et al., (2016), eles são excelentes opções para uso nesses sistemas, sendo que a grama esmerada é a mais utilizada em todo o Brasil (GODOY et al., 2012), e sua utilização como cobertura vegetal em telhados verdes vem crescendo nos últimos anos (ARAUJO, 2011).

Assim, áreas gramadas apresentam elevada importância na implantação de projetos de paisagismo, contudo, conhecer melhor a dinâmica de cada espécie e sua manutenção no decorrer no tempo, se faz essencial para um melhor estudo de seu uso na arquitetura paisagística.

\section{CARACTERÍSTICAS ADAPTATIVAS DAS GRAMAS}

As gramas pertencem à família das gramíneas, Poaceae (Gramineae), e são mais de 10000 espécies, porém menos de 50 são utilizadas para a formação de gramados (WATSON; DALLWITZ, 1992). 
Em relação ao clima, as gramas são divididas em dois grupos: de clima quente e de clima frio (BEARD, 1973). Porém, segundo Gurgel (2003), no Brasil são cultivadas somente gramas de clima quente, como a Esmeralda ou Japonesa (Zoysia japonica Steud.), a Santo Agostinho ou Inglesa (Stenotaphrum secundatum Kuntze), a Bermuda (Cynodon dactylon L.) e a São Carlos (Axonopus affinis Chase).

Segundo Godoy et al. (2012), as gramas de clima quente são separadas em dois grupos: rizomatosas, onde a base do desenvolvimento vegetativo se dá a partir de rizomas subsuperficiais e, portanto, podem ser colhidas em área total visto que, após a colheita, estes rizomas são capazes de brotar e cobrir novamente o solo; e estoloníferas, onde a base do desenvolvimento vegetativo se dá a partir de estolões superficiais e, dessa forma, no momento da colheita tem-se a necessidade de deixar faixas para que ocorra a formação de um novo tapete.

As variedades rizomatosas possuem alta capacidade de regeneração, principalmente se a injúria for causada por tráfego excessivo, isto ocorre porque os rizomas estão enterrados em subsuperfícies e, por isso, ficam protegidos de danos mecânicos diretos. Porém, são variedades altamente exigentes em manutenção, desde adubação até poda, havendo algumas situações em que a poda deve ser diária (GURGEL, 2003); elas crescem em alta densidade, sob contínua desfolhação provocada pelo corte (CHRISTIANS, 1998). Localizado na base da planta, formado por um grupo de nós e entrenós compactados, fica o meristema subapical da grama, principal motivo pela qual as gramas podem receber podas continuamente (UNRUH, 2004). Neste grupo se encaixam as variedades de bermudas, Zoysias e Seashore paspalum.

De acordo com Godoy et al. (2012), as estoloníferas não possuem boa capacidade de suportar tráfego intenso, pois como apresentam estolões superficiais, estes podem ser danificados. Como geralmente possuem folhas largas, auxiliando na interceptação de luz para a fotossíntese, desenvolvem-se bem em áreas sombreadas (UNRUH, 2004); Santo Agostinho e São Carlos são exemplos de estoloníferas.

GRAMA ESMERALDA (Zoysia japonica Steud.) (GODOY; VILLAS BÔAS, 2008).

A grama esmeralda é originária da Ásia e foi introduzida no Brasil no início da década de 1980. É a grama mais comercializada no Brasil entre as gramas cultivadas, desde a região sul até a região nordeste. 
É uma planta de ciclo perene de crescimento prostrado, de altura baixa, rizomatosa. Suas folhas são enroladas na gema, possuem textura média e lígula com uma franja de pelos, estreitas e rígidas, de coloração verde de intensidade média. $\mathrm{O}$ ápice da folha é pontiagudo, ficando com o aspecto de agulha quando em estresse hídrico. A inflorescência é uma única espiga com espiguetas acuminadas alternando ao longo da ráquis.

Essa grama possui ampla adaptação às condições brasileiras, podendo ser usada de Norte a Sul, em regiões litorâneas ou nos Planaltos. Devido a sua boa capacidade de resistência ao pisoteio foi, durante muitos anos, a variedade dos principais gramados de futebol do Brasil. Também é a espécie de grama da grande maioria dos gramados residenciais brasileiros. Devido ao seu forte sistema radículas e rizomas, é uma grama bastante usada em contensão de taludes e em áreas de potenciais problemas de erosão. Adapta-se a diferentes tipos de solo, desde arenosos a argilosos, exceto aos solos com baixa capacidade de drenagem. Desenvolve-se bem em áreas de plena insolação, tolerando um mínimo de sombreamento.

\section{IMPLANTAÇÃO DE GRAMADOS ORNAMENTAIS}

São recomendadas quatro formas de implantação para gramados ornamentais: placas, tapetes, rolos e plugs (LIMA et al., 2010).

O uso de placas e tapetes de grama é uma forma rápida e eficiente para a implantação de gramados em área não muito grande, como residências e comércio. A dimensão das placas é de $30 \mathrm{~cm} \times 30 \mathrm{~cm}$ e dos tapetes varia de $40 \mathrm{~cm} \times 125 \mathrm{~cm}$ ou $40 \mathrm{~cm} \times 2,5 \mathrm{~cm}$ (RAATS et al., 2012). O rolo, recomendado para grandes gramados, como campos esportivos e clubes, tem $0,75 \mathrm{~m}$ de largura por 40 a $60 \mathrm{~m}$ de comprimento, e com o seu uso é possível plantar até $5000 \mathrm{~m}^{2}$ de grama um único dia (GODOY; ALMEIDA, 2015).

Os plugs são pequenas mudas de gramas enraizadas, produzidas em bandejas descartáveis (RAATS et al., 2012), como mostrado na Figura 1a. De acordo com Pimenta (2003), existem dois tipos de plugs: plugão, dispostos em bandejas de 35 unidades, que servem para o plantio desmembrado de até $6 \mathrm{~m}^{2}$; e o pluguinho, disposto em bandejas de 64 unidades, servindo para o plantio desmembrado de até $8 \mathrm{~m}^{2}$. A sua produção é similar à produção de outras mudas de plantas que se proliferam vegetativamente. São de baixo custo de implantação e formação de um gramado, sendo comprovada uma 
redução de até um terço no capital envolvido. O tempo de formação de um gramado implantado por plugs (Figura 1b) varia de 3 a 8 meses (RAATS et al., 2012).
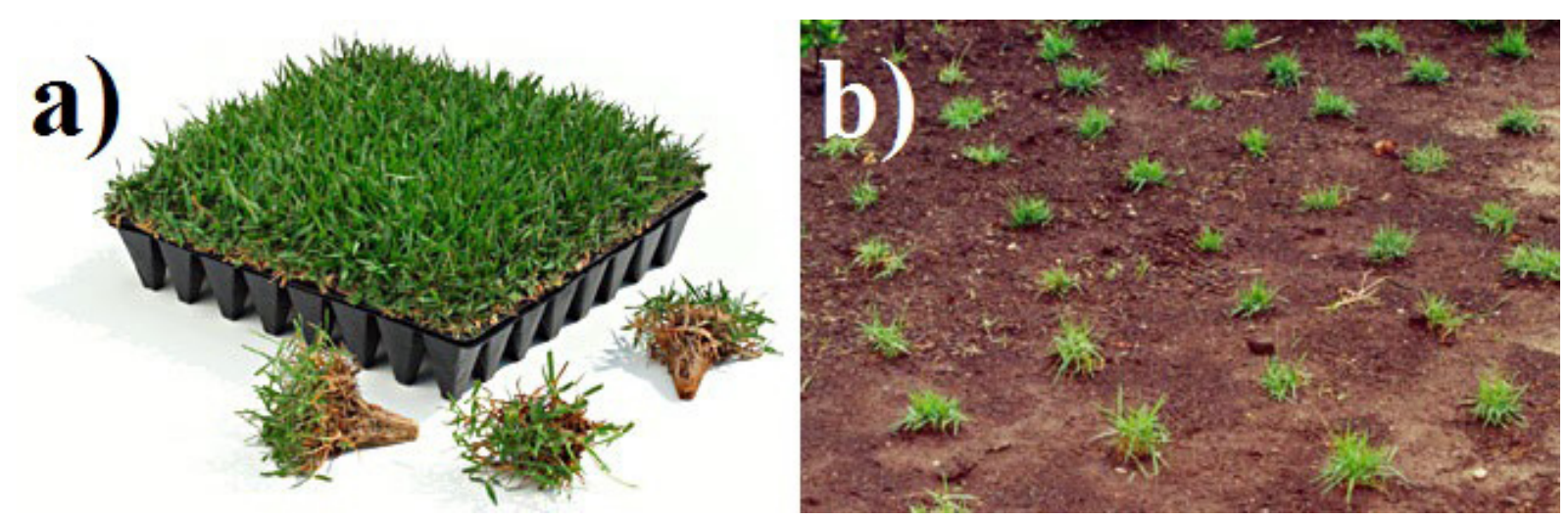

Figura 1 - Plugs dispostos em bandeja (a) e plugs implantados em jardim (b). Fonte: Itograss, 2018

Para Brito (2010) há algumas etapas que devem ser seguidas no que se diz respeito a implantação de um gramado, sendo elas:

\section{Preparo do terreno}

Em primeiro lugar, deve-se eliminar as plantas daninhas, para que não haja competição entre elas e o gramado, com capina manual ou mecânica (BRITO, 2010). Geralmente, a capina manual basta, porém, quando trata-se do aparecimento de plantas como tiririca (Cyperus rotundus) ou braquiária (Brachiaria spp.), a eliminação com produtos químicos é recomendada (MACIEL, 2010).

Caso seja em uma construção nova, após a eliminação de plantas daninhas, deve ser feita uma raspagem geral do terreno, para eliminar todos os restos da construção como massa, madeiras, pedras, restos de cal e cimento. Esses tipos de materiais não devem ser depositados antes para a colocação da terra, pois geram gramas de péssima qualidade e desuniforme (BRITO, 2010). O preparo do solo é a etapa mais importante, pois garante boa formação das raízes, maciez e oxigenação do solo (GODOY et al., 2012).

De acordo com Godoy et al., (2007) é indicado que se faça a escarificação do solo, pois a falta dela causa prejuízos na formação das raízes da grama. Um solo ideal deve possuir a parte sólida, espaço poroso e água; e na ausência da escarificação, o solo 
fica compactado e não permite troca gasosa, os espaços para a retenção de água ficam comprometidos.

O excesso de umidade acarreta em diminuição da oxigenação. Isso acontece quando há escoamento superficial de água da construção sobre o gramado, fazendo com que haja empoçamento de água da chuva. Para evitar formação de caminhos de água deve ser feito um nivelamento do terreno, aplainando o solo com uma régua de madeira e eliminando todas as imperfeições do terreno (BRITO, 2010).

\section{Aplicação de fertilizantes}

É importante que seja feita a aplicação de fertilizantes para repor os nutrientes que estão presentes em baixa quantidade no solo, garantindo bom enraizamento do gramado e formação de folhas (CASTILHO; MATEUS, 2003).

Ainda, é necessário que seja feito uma análise química do solo para saber quais nutrientes serão aplicados e em que quantidades eles serão aplicados, para evitar a falta ou o excesso dos mesmos (GODOY et al., 2012). A aplicação pode ser manual ou através de equipamentos.

A distribuição de calcário e dos fertilizantes deve ser feita antes da escarificação, para serem bem incorporados ao solo, permitindo a correção da fertilidade do solo em profundidade, garantindo a formação de raízes mais profundas (BRITO, 2010).

\section{Cuidados e colocação do gramado}

A grama deve ser comprada pouco antes de ser implantada, pois não deve ficar empiIhada, podendo fazer com que a mesma fermente, acarretando em morte de parte dos tapetes ou dos tapetes inteiros, provocando um péssimo resultado no acabamento final (MARTELLO et al., 2014).

Quando for realizado o assentamento dos tapetes, deve-se disfarçar ao máximo a parte onde estão encostados entre si, acondicionando uma fileira de tapetes intercalada a outra fileira subsequente (BRITO, 2010). É indicado o uso de soquete ou rolete para melhorar a fixação do tapete sobre o solo. 
É importante preencher todos os rejuntes dos tapetes logo após o seu plantio com uma mistura de areia fina com substrato, para impedir a entrada de luz do sol, evitando que sementes que já estejam no solo germinem (BRITO, 2010).

De acordo com Carribeiro (2010) para que se tenha um bom pegamento dos tapetes e que fique sempre verde é necessário irrigar. Por isso, recomenda-se a utilização de aspersor fixo ou móvel, lembrando que este segundo, deve ser trocado de lugar para que todas as partes do gramado sejam atingidas. Deve-se tomar cuidado com o encharcamento, pois compromete a qualidade de formação do gramado. Para a manutenção do gramado é necessário que se realize podas, adubação de cobertura e irrigação (AMARAL; CASTILHO, 2012).

\section{MATERIAL E MÉTODOS}

\section{Localização}

O presente trabalho foi realizado no município de llha Solteira-SP (coordenadas geográficas $51^{\circ} 06^{\prime} 35^{\prime \prime}$ Oeste e $20^{\circ} 39^{\prime} 44^{\prime \prime}$ Sul). Os locais estudados foram: duas residências, uma localizada na zona sul (R1) e outra na zona norte (R2) e uma instituição de ensino particular, localizada no centro (E) (Figura 2), sendo que todos os locais se encontravam implantados com gramado ornamental de grama esmeralda.
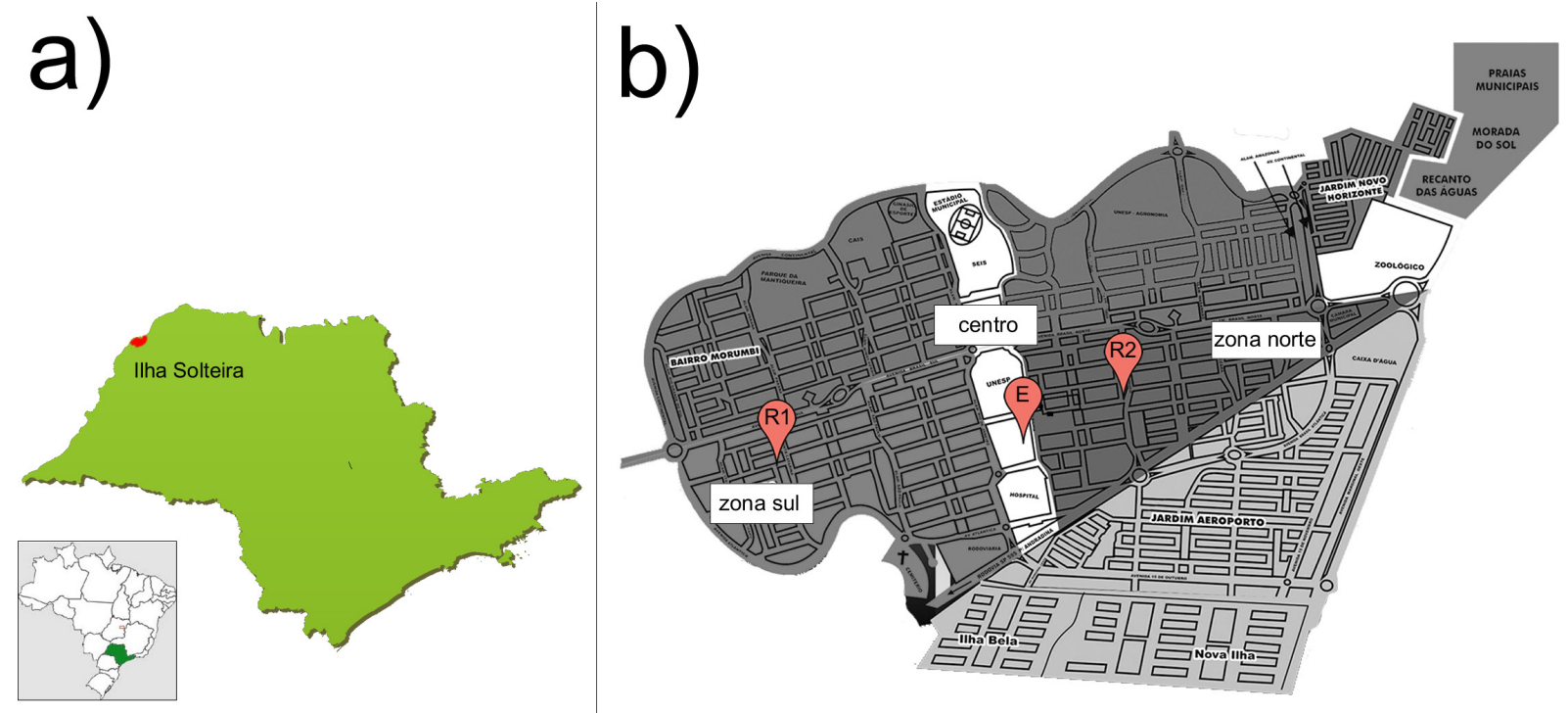

Figura 2 - (a) Localização da cidade de llha Solteira/SP (REDE CIDADE DIGITAL, 2018). (b) Mapa da cidade de Ilha Solteira e locais estudados (ILHA SOLTEIRA, 2018, adaptado) 
Na residência R1, o gramado foi implantado em 1998; e em R2 e E, setembro de 2001, utilizando-se tapetes; todos são irrigados quando necessário.

As coletas de solo foram realizadas em 2016, nos dias 19 de maio, para R1 e 23 de maio para R2 e E.

\section{Avaliações}

Índice de conteúdo de clorofila e análise foliar (ICC): foram determinados com o auxílio de clorofilômetro manual - Chlorophyll Content Meter (CCM 200). Foram analisados 3 pontos em cada local (Figura 3), sendo que em cada ponto foram feitas 3 medições - sendo coletadas 3 folhas da grama de cada ponto, estas dispostas no clorofilômetro de modo a cobrir inteiramente o detector, sem que as folhas fossem sobrepostas, sendo realizada três leituras por metro quadrado $(C 1, C 2, C 3 ; C 4, C 5$, C6; C7, C8, C9) -totalizando 9 leituras.

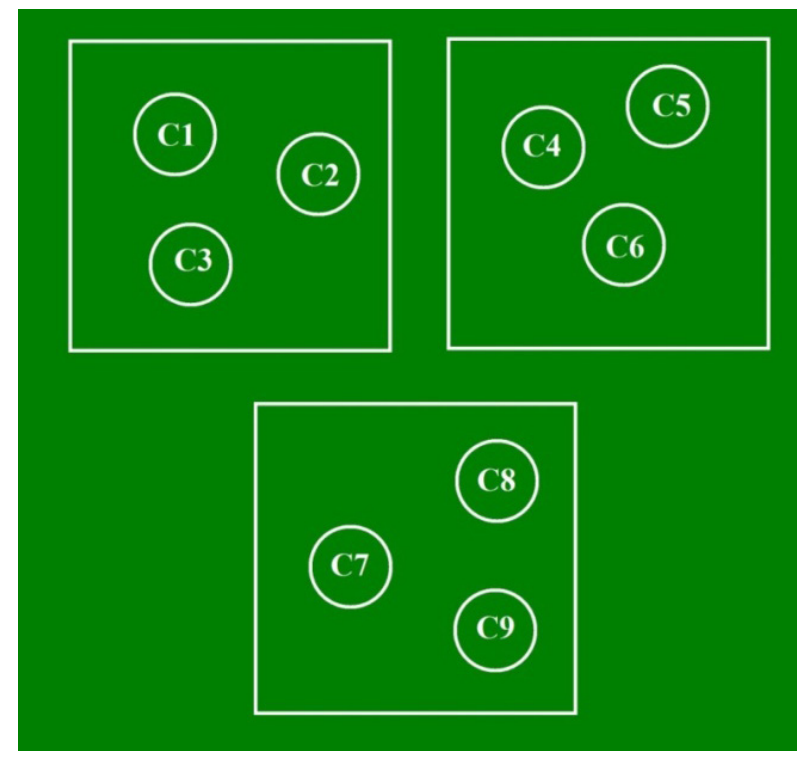

Figura 3 - Pontos utilizados para determinação do teor de clorofila e análise foliar. Fonte: próprios autores

Análise do estado nutricional do gramado: Após leitura do ICC, foram coletadas folhas dos mesmos pontos e colocadas em papel pardo e levadas para secar na estufa a $25^{\circ} \mathrm{C}$, por 24 horas. Depois disso, foram moídas e preparadas para análise química do tecido vegetal. Esta técnica permite quantificar a concentração dos nutrientes e por meio desta quantificação estimar o estado nutricional da planta. A análise foliar de nitrogênio foi efetuada pelo método semi-micro Kjeldahl, após digestão sulfúrica (MALAVOLTA et al., 1997). 


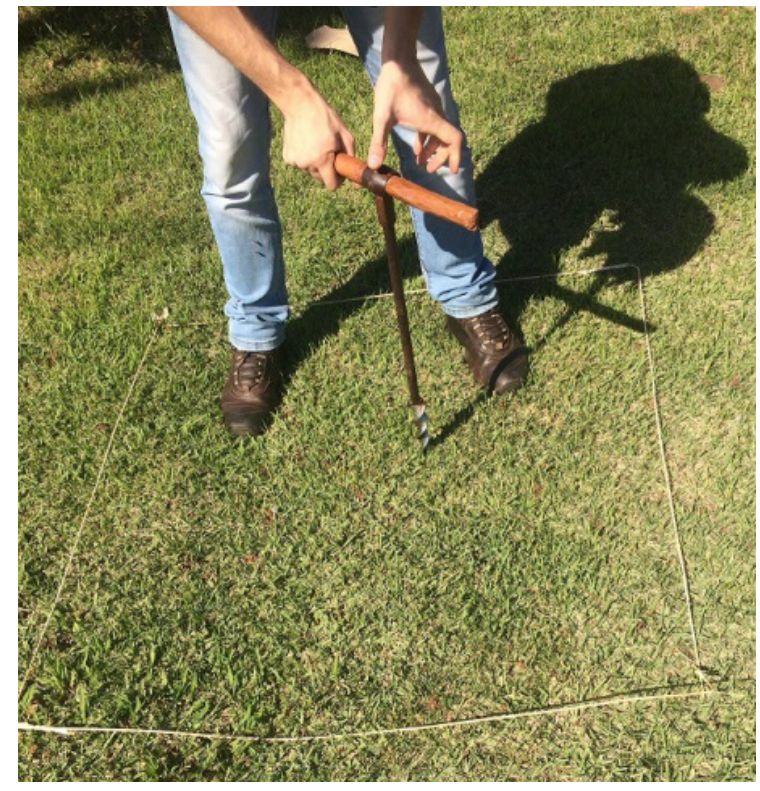

Análise química do solo: Para cada local foram retiradas amostras de três pontos, escolhidos aleatoriamente, dentro de um metro quadrado cada (Figura 4).

Para a análise química do solo, em cada quadrado foram recolhidas três amostras de solo (S1, S2 e S3), que foram homogeneizadas e submetidas a análise química, segundo Raij et al. (2001), no Laboratório de Fertilidade do Solo da UNESP, Campus de Ilha Solteira/SP.

Figura 4 - Coleta para posterior análise de solo.

Fonte: próprios autores.

\section{RESULTADOS E DISCUSSÃO}

\section{Análise do estado nutricional e Índice de Conteúdo de Clorofila}

Na Tabela 1 constam os dados de teores de nutrientes e ICC em folhas de grama esmeralda.

Tabela 1 - Valores médios dos teores de nutrientes e ICC em folhas de grama esmeralda, Ilha Solteira, 2016.

\begin{tabular}{cccccccccccc}
\hline & $\mathbf{N}$ & $\mathbf{P}$ & $\mathbf{K}$ & $\mathbf{C a}$ & $\mathbf{M g}$ & $\mathbf{S}$ & $\mathbf{C u}$ & $\mathbf{F e}$ & $\mathbf{M n}$ & $\mathbf{Z n}$ & $\mathbf{I C C}$ \\
Trat. & & & & & & & & & & \\
\hline R1 & 25,27 & 19,29 & 10,47 & 1,93 & 1,83 & 7,77 & 13,00 & 366,33 & 131,33 & 26,33 & 36,19 \\
R2 & 17,24 & 26,58 & 9,96 & 0,70 & 1,09 & 4,21 & 25,33 & 340,33 & 59,67 & 25,00 & 19,96 \\
E & 22,80 & 25,36 & 16,60 & 1,31 & 1,74 & 5,21 & 10,33 & 259,00 & 39,00 & 26,33 & 19,79 \\
\hline
\end{tabular}

Mills e Jones (1996) dizem que valores ideais de $\mathrm{N}$ são aqueles compreendidos entre 20 e $24 \mathrm{~g} / \mathrm{kg}$, onde somente R1 ultrapassa esse valor, chegando a atingir 25,27 g/ $\mathrm{kg}$. 
Para Godoy e Villas Bôas (2010) dizem que o ideal está na faixa de 14 a $25 \mathrm{~g} / \mathrm{kg}$; aqui, somente R1 não se encaixa nessa faixa, sendo apenas $0,27 \mathrm{~g} / \mathrm{kg}$ acima do valor ideal. Piedade (2004) afirma que valores entre 17 e $21 \mathrm{~g} / \mathrm{kg}$ são ótimos; e de acordo com essa faixa, somente R2 se encaixa, apresentando um valor de 17,24 g/kg. Os locais R1 e E apresentam maiores valores de $\mathrm{N}$ foliar, sendo $25,27 \mathrm{~g} / \mathrm{kg}$ e $22,80 \mathrm{~g} / \mathrm{kg}$, respectivamente (Tabela 1), sendo que para Carribeiro (2010), os valores ideais estão entre 15 e $20 \mathrm{~g} / \mathrm{kg}$ de $\mathrm{N}$ foliar, onde somente R2 se encaixa nesses valores; os locais R1 e E estão acima do intervalo citado pelo autor.

Em trabalho realizado por Santos e Castilho (2015) com grama esmeralda os valores de $\mathrm{N}$ encontrados foram entre 14,03 e 17,68 g/kg, inferiores a R1 e E.

O N foliar é influenciado pela quantidade de P no solo. Quando este está baixo (Tabela 2), o $\mathrm{N}$ foliar também é baixo (Tabela 1), porque suprimentos inadequados de $\mathrm{P}$ podem diminuir a absorção de $\mathrm{NO}_{3}$ e $\mathrm{NH}_{4}$. R1 é o local que apresenta maiores valores de $\mathrm{P}$ no solo (Tabela 2) e, portanto, maior $\mathrm{N}$ foliar (Tabela 1).

Maiores teores de $\mathrm{N}$ e Mg na folha, refletem num maior ICC (Tabela 1), pois de acordo com Taiz e Zeiger (2017) as clorofilas, são moléculas formadas por um átomo central de magnésio, ligado a 4 outros de nitrogênio. Assim, existe uma correlação entre as concentrações de clorofila e o estado nutricional da planta (SANTOS; CASTILHO, 2015) como é perceptível no local R1. Isso também pode estar refletindo na quantidade de Mg no solo, visto na Tabela 2. Nos locais R2 e E, que apresentam menor quantidade de $\mathrm{N}$ e $\mathrm{Mg}$ foliar, apresenta também menor ICC e isso acontece porque a molécula de clorofila apresenta $\mathrm{N}$ e Mg em sua composição.

Os valores encontrados em N para R2, K para R1 e R2, Ca e Zn para todos os locais, e Mg em R2 são considerados abaixo do adequado segundo Mills e Jones (1996) para o gênero Zoysia. Por outro lado, para Jones (1980), os valores de N, K, Ca e Mg estão abaixo do considerado adequado para espécies de gramas utilizadas nos EUA.

Lima et al. (2008), trabalhando com grama esmeralda, afirma que o índice de clorofila encontrado nos resultados é indicativo do estado nutricional em $\mathrm{N}$, e quando os autores, usando diferentes doses de fonte de $\mathrm{N}$, verificaram aumento nas leituras em clorofilômetro em decorrência do aumento dos teores de $\mathrm{N}$, conferindo maior intensidade de cor verde e maior concentração de N, em um intervalo de 36,5 a 39,2 ICC; no presente trabalho, nenhum dos tratamentos atingiu o referido valor, porém R1 apresenta-se pró- 
ximo deste intervalo (36,19 ICC). Dinalli (2012), assim como Santos e Castilho (2015), observaram valores no intervalo de 17,9 a 19,4 ICC e de 14,95 a 19,54, respectivamente, sendo esses menores do que os encontrados no presente trabalho.

Santos et al., (2016), avaliando a influencia de diferentes substratos no teor de clorofila das folhas de grama esmeralda, encontraram intervalos entre 13,09 a 18,18 CCl, também inferiores aos do estudo.

\section{Análise de solo}

Na Tabela 2 são apresentados os resultados analíticos dos solos encontrados nos locais avaliados.

Tabela 2 - Resultados analíticos dos solos. Ilha Solteira, 2016.

\begin{tabular}{|c|c|c|c|c|c|c|c|c|c|c|}
\hline Locais & $\begin{array}{c}\mathbf{p H} \\
\mathrm{CaCl}_{2}\end{array}$ & $\begin{array}{c}\mathbf{P} \\
\mathrm{mg} / \mathrm{dm}^{3}\end{array}$ & $\begin{array}{c}\text { MO } \\
\mathrm{g} / \mathrm{dm}^{3}\end{array}$ & K & Ca & Mg & $\begin{array}{c}\mathrm{H}+\mathrm{Al} \\
\mathrm{nmolc} / \mathrm{d}\end{array}$ & Al & SB & CTC \\
\hline $\mathrm{R} 1$ & 6,0 & 37 & 33 & 1,0 & 64 & 14 & 18 & 0 & 79,0 & 97,0 \\
\hline $\mathrm{R} 2$ & 5,7 & 16 & 26 & 1,4 & 31 & 9 & 16 & 0 & 41,4 & 57,4 \\
\hline $\mathrm{E}$ & 6,0 & 18 & 20 & 1,7 & 28 & 10 & 13 & 0 & 39,7 & 52,7 \\
\hline Locais & V & $\mathrm{Ca} / \mathrm{CTC}$ & Mg/CTC & Sat. Al & $\mathbf{S}$ & B & $\mathrm{Cu}$ & $\begin{array}{l}\mathrm{Fe} \\
y / \mathrm{dm}^{3}\end{array}$ & $M n$ & $\mathrm{Zn}$ \\
\hline $\mathrm{R} 1$ & 81 & 66 & 14 & 0 & 4 & 0,10 & 0,9 & 31 & 17,4 & 2,5 \\
\hline $\mathrm{R} 2$ & 72 & 54 & 16 & 0 & 4 & 0,10 & 1,8 & 32 & 18,6 & 2,8 \\
\hline$E$ & 75 & 53 & 19 & 0 & 3 & 0,06 & 0,8 & 5 & 4,0 & 2,7 \\
\hline
\end{tabular}

Valores de pH entre 5,5 e 6,5 são considerados aceitáveis para gramas, de acordo com Christian (1998) e, segundo Carrow et al (2001), a grama esmeralda é tolerante a alta acidez do solo ( $\mathrm{pH}$ em água $<5,0$ ou $\mathrm{pH}$ em $\mathrm{CaCl}_{2}<4,4$ ), sendo que no presente trabalho todos os valores são superiores ao citado por esse autor, e dentro da faixa considerada por Christian (1998). Porém, como no Brasil há solos mais intemperizados e que sofrem alteração da CTC com a variação do $\mathrm{pH}$, a dose de corretivo a ser utilizada é baseada na saturação por bases do solo (V\%) que se correlaciona diretamente com o valor de pH na solução do solo. Santos e Castilho (2016) com 
caracterização química de substratos para desenvolvimento da grama esmeralda, observaram um intervalo variando de 4,5 a 7,5, e constataram que com o maior valor de $\mathrm{pH}$, melhor foi o cultivo do gramado.

Com relação ao P, de acordo com Christian (1998), para grama esmeralda, teores maiores que $20 \mathrm{mg} / \mathrm{dm}^{3}$ são considerados alto, sendo que somente R1 apresenta esse resultado. $\mathrm{R} 2 \mathrm{e} \mathrm{E}$, segundo o mesmo autor, possuem teor médio de $\mathrm{P}$ (entre $10 \mathrm{e}$ $20 \mathrm{mg} / \mathrm{dm}^{3}$ ). Godoy e Villas Bôas (2003) afirmam que teores acima de $15 \mathrm{mg} / \mathrm{dm}^{3}$ de P no solo podem ser considerados adequados para gramados, portanto, todos os locais se encaixam nesse valor.

Segundo Raij (2011), valores acima de $7 \mathrm{mmolc} / \mathrm{dm}^{3}$ e $8 \mathrm{mmolc} / \mathrm{dm}^{3}$ são considerados altos para $\mathrm{Ca}$ e $\mathrm{Mg}$, respectivamente. Altas quantidades de $\mathrm{Ca}$ e $\mathrm{Mg}$ podem estar relacionadas as baixas quantidades de $\mathrm{K}$, como as encontradas em R1 e R2. O local E possui teor médio de K (Tabela 2), possivelmente por apresentar menor quantidade de Ca do que os demais locais. Por outro lado, Godoy e Villas Bôas (2003), trabalhando com grama esmeralda, afirmam que teores de $\mathrm{K}$ entre $1,1-4,5 \mathrm{mmolc} / \mathrm{dm}^{3}$ são considerados baixos.

O acúmulo de matéria orgânica fornece aumento expansivo da CTC, como pode ser visto em R1, que foi o de maior quantidade de matéria orgânica (33 g/dm ${ }^{3}$ ) e, consequentemente, o que apresentou a maior CTC $\left(97 \mathrm{mmolc} / \mathrm{dm}^{3}\right)$.

De acordo com os valores mostrados na Tabela 2 e quando comparados a Tabela 3, nota-se que em relação ao $\mathrm{B}$ e $\mathrm{Cu}$, todos os resultados obtidos nos locais estudados são menores; para Fe e Mn são menores somente no local E; e para Zn, são maiores.

Tabela 3 - Teores médios de micronutrientes encontrados em áreas de produção de gramas antes da instalação dos experimentos (camada de 0 a $20 \mathrm{~cm}$ ) nos municípios de Capela do Alto e Itapetininga/SP. Adaptado de Lima (2009), Backes (2008) e Godoy (2005).

\begin{tabular}{ccccc}
\hline \multicolumn{5}{c}{ Micronutrientes } \\
\hline B & Cu & Fe & Mn & Zn \\
& & & & \\
\hline 0,23 & 2,4 & 25,3 & 4,3 & 0,3 \\
\hline
\end{tabular}




\section{Necessidade de Calagem}

Segundo Godoy e Villas Bôas (2008), no Brasil, o método que mais se utiliza para determinar a quantidade de corretivo necessária é o baseado na saturação por bases do solo (V\%), a qual se correlaciona diretamente com o valor de pH da solução do solo, valores demonstrados na Tabela 4.

Tabela 4 - Relação entre a saturação por bases (V\%), pH em água e em $\mathrm{CaCl}_{2}$ do solo. Adaptado de Quaggio et al. (1982).

\begin{tabular}{ccc}
\hline Saturação por bases (V\%) & $\mathbf{p H}$ em água ${ }^{1}$ & $\mathbf{p H ~ e m ~ C a C l}_{2}{ }^{2}$ \\
\hline 30 & 5,1 & 4,5 \\
40 & 5,3 & 4,7 \\
50 & 5,5 & 5,0 \\
60 & 5,7 & 5,3 \\
70 & 5,9 & 5,6 \\
80 & 6,0 & 5,8 \\
\hline
\end{tabular}

${ }^{1}$ calculado com base na diferença de 0,6 unidades a menos que o $\mathrm{pH}$ em $\mathrm{CaCl}_{2} ;{ }^{2} \mathrm{pH}\left(\mathrm{CaCl}_{2}\right)=3,66+0,27 \mathrm{~V}$ **$(\mathrm{r}=0,97)$

A Tabela 5 apresenta os valores de pH, saturação por bases e CTC dos locais estudados para este trabalho.

Tabela 5 - Valores de pH, saturação por bases (V\%) e capacidade de troca catiônica (CTC) dos locais estudados.

\begin{tabular}{cccc}
\hline Locais & $\mathbf{p H}\left(\mathbf{e m ~} \mathrm{CaCl}_{2}\right)$ & $\mathbf{V}(\%)$ & $\mathbf{C T C}\left(\mathbf{m m o l c} / \mathbf{d m}^{3}\right)$ \\
\hline $\mathrm{R} 1$ & 6,0 & 81 & 97 \\
$\mathrm{R} 2$ & 5,7 & 72 & 57,4 \\
$\mathrm{E}$ & 6,0 & 75 & 52,7 \\
\hline
\end{tabular}

De acordo com a Tabela 2 e os resultados apresentados nos locais R1 $(\mathrm{V} \%=81)$ e R2 $(V \%=72)$ não há necessidade de calagem. Já o local $E$ necessita e esta pode ser calculada pela seguinte fórmula:

$$
Q C=\frac{[(V 2-V 1) \times C T C]}{P R N T \times 10} \times f
$$


Sendo:

$\mathrm{QC}=$ quantidade de corretivo a ser aplicado, em toneladas por hectare (t há-1);

V2 = saturação por bases ideal para a espécie de grama;

V1 = saturação por bases do solo na camada que se deseja corrigir;

CTC = capacidade de troca catiônica do solo, em $\mathrm{mmol}_{\mathrm{C}} \mathrm{dm}^{-3}$;

PRNT = Poder Relativo de Neutralização Total do corretivo, em \%;

$\mathrm{F}=$ fator de correção para a profundidade de incorporação do corretivo. Utilizar $f=0,5$ para camada de 0 a $0,1 \mathrm{~m} ; f=1$ para camada de 0 a $0,2 m ; f=1,5$ para camada de 0 a $0,3 \mathrm{~m}$.

Para o local E, a quantidade de corretivo a ser aplicada, de acordo com a fórmula dada, seria de 0,33 t/ha.

Porém, de acordo com Godoy (2005), para a produção de tapetes de grama esmeralda em solo muito argiloso no município de Itapetininga - SP, avaliando doses de calcário calculadas para elevar a saturação por bases do solo a 45, 60 e 75\% na camada de 0 a 0,1 m e na camada de 0 a 0,2 m, mais um tratamento controle que não foi utilizado calcário, observaram que a parcela que não recebeu calcário formou tapete de grama no mesmo tempo que as gramas que receberam a maior dose de calcário.

Para não haver problemas de deficiência de $\mathrm{Ca}$ e $\mathrm{Mg}$ ou toxicidade por $\mathrm{Al}$ recomenda-se a utilização do valor de saturação por bases de $70 \%$ para implantação de gramados e em áreas de início de produção de tapetes e de $60 \%$ para gramados implantados (VILLAS BÔAS; GODOY, 2008). De acordo com esses dados, nenhum dos locais necessitaria de correção de calagem, tendo em vista que as saturações por bases são maiores do que $60 \%$.

\section{Necessidade de Gessagem}

O gesso agrícola deve ser aplicado quando pelo menos uma dessas condições seja satisfeita: o teor de cálcio deve ser menor ou igual a $4 \mathrm{mmolc} / \mathrm{dm}^{3}$; o teor de alumínio maior que $5 \mathrm{mmolc} / \mathrm{dm}^{3}$; a saturação por alumínio ser maior que $30 \%$. 
Na Tabela 6 estão presentes os valores de Ca, Al e saturação por alumínio dos gramados estudados.

Tabela 6 - Valores de cálcio, alumínio e saturação por alumínio ( $m \%$ ) dos locais estudados.

\begin{tabular}{cccc}
\hline Locais & Ca & Al & $\begin{array}{c}\text { Sat. por Al } \\
\text { m\% }\end{array}$ \\
\hline R1 & 64 & 0 & 0 \\
R2 & 31 & 0 & 0 \\
E & 28 & 0 & 0 \\
\hline
\end{tabular}

De acordo com os dados obtidos no Laboratório de Fertilidade de Solos da UNESP de Ilha Solteira (Tabela 6), nenhum desses requisitos se encaixa nos solos estudados. E por isso, o solo não necessita de gessagem.

\section{Recomendação de adubação}

Os locais estudados não necessitaram de adubação e, segundo Coutinho (2004), isso aconteceu porque a quantidade de nutrientes disponíveis nos restos de podas, seja do gramado ou proveniente do paisagismo, pode voltar para auxiliar na nutrição do gramado novamente, além de manter a flora microbiana benéfica no solo, reduzindo o aparecimento de pragas e doenças.

\section{CONCLUSÃO}

Os gramados avaliados se encontravam em boas condições, baseado nos resultados de análise de solo e do estado nutricional das folhas, mostrando que em um projeto paisagístico de implantação de um gramado ornamental, este pode durar por muitos anos, sem necessidade de renovação da espécie vegetal.

O clorofilômetro portátil pode ser uma excelente alternativa para avaliar indiretamente a nutrição de uma área gramada, principalmente para determinação de nitrogênio, assim empresas de manutenção paisagística, podem saber com melhor exatidão a hora necessária para adubação. 
Nos locais estudados, com grama esmeralda, verificou-se que não é necessária adubação de manutenção, provavelmente devido aos restos de poda possuírem uma boa quantidade de nutriente que são reciclados, principalmente referentes ao nitrogênio e magnésio que fazem parte da molécula de clorofila. Dessa forma, áreas gramadas são excelentes opções para uso em projetos sustentáveis, por necessitarem menor gastos com fertilizantes químicos

\section{REFERÊNCIAS BIBLIOGRÁFICAS}

AMARAL, J. A.; CASTILHO, R. M. M. Fertilizantes comerciais de liberação imediata e controlada na revitalização de grama batatais. Revista Científica Eletrônica de Agronomia, Garça, v. 22, n. 2, p.1-11, 2012.

ANDRADE, P. F. S. Análise da conjuntura agropecuária. Safra 2015/2016: Estado do Paraná. Secretaria da agricultura e do abastecimento. Departamento de Economia Rural. 2016. Disponível em: <http://www.agricultura.pr.gov.br/arquivos/File/deral/ Prognosticos/2016/flores_2015_16.pdf>. Acesso em: 08 dez. 2016.

ANGELIS, B. L. D.; PEREIRA, A. D.; ANGELIS NETO, G.; BARROS, R. A. A função das áreas verdes em parques industriais: o caso de Maringá, Estado do Paraná. Acta Sci. Technol. v. 29, n. 2, p. 187-193, 2007.

ARAÚJO, S.R. As funções dos telhados verdes no meio urbano, na gestão e no planejamento de recursos hídricos. Monografia (Engenharia Florestal) - Instituto de Florestas/Universidade Federal Rural do Rio de Janeiro, Seropédica/RJ, 2007. 28 p.

BACKES, C. Aplicação e efeito residual do lodo de esgoto em sistemas de produção de tapetes de grama esmeralda. 2008. 152 f. Tese (Doutorado em Agronomia/ Horticultura) - Faculdade de Ciências Agronômicas, Universidade Estadual Paulista, Botucatu, 2008.

BEARD, J. B. Cool season turfgrasses. Turfgrass: Science and Culture. Englewood Cliffs, NJ: Prentice-Hall, 1973, p. 54-131.

BRITO, J. F. F. Erros na implantação e manutenção de gramados ornamentais. In: GODOY; L. J. G.; MATEUS, C. M. D.; BACKES, C.; VILLAS BÔAS, R. L. Tópicos atuais em gramados II. Ed. FEPAF, Botucatu p. 103-114, 2010. 
CARRIBEIRO, L. S. Potencial de água no solo e níveis de compactação para o cultivo de grama esmeralda. 2010. 74 f. Dissertação (Mestrado em Agronomia/Irrigação e Drenagem) - Faculdade de Ciências Agronômicas, Universidade Estadual Paulista, Botucatu, 2010.

CARROW, R. N.; WADDINGTON, D. V.; RIEKE, P. E. Turfgrass soil fertility and chemical problems: Assessment and management. Chelsea Ann Arbor Press, 2001. 400 p.

CASTILHO, R. M. M.; MATHEUS, C. M. D. Calagem e Adubação para gramados ornamentais. In: III Sigra - Simpósio sobre gramados, 3. Botucatu, 2006. 6p. (CD-ROM).

CHRISTIANS, N. E. Fundamentals of turfgrass management. Chelsea Arbor Press, 1998. 301p.

COUTINHO, A. A. Experiência com aproveitamento dos resíduos do corte de grama. In: II SIMPÓSIO SOBRE GRAMADOS - SIGRA, 2. Botucatu, 2004. 18p. (CD-ROM).

DINALLI, R. P.; BUZETTI, S.; CASTILHO, R. M. M.; GAZOLA, R. N.; CELESTRINO, T. $S$. Índice de clorofila na folha de grama esmeralda em função da aplicação de fontes de N. In: VI SIGRA - Simpósio de Gramados - Anais. UNESP - Faculdade de Ciências Agronômicas, Botucatu, SP, 15 e 16 de maio de 2012.

GODOY, L. J. G. Adubação nitrogenada para produção de tapetes da grama Sto. Agostinho e Esmeralda. 2005. Tese (Doutorado em Agronomia/Agricultura) - Faculdade de Ciências Agronômicas, Universidade Estadual Paulista, Botucatu, 2005.

GODOY, L. J. G. de; VILLAS BÔAS, R. L.; BACKES, C.; SANTOS, A. J. M. Nutrição, Adubação e Calagem para produção de gramas. 1 Ed. FEPAF, 2012. 146p.

GODOY, L. J. G.; ALMEIDA, L. C. F. Sazonalidade e evolução dos preços da grama esmralda, no ceasa campinas-SP, no período de 2010 a 2015. In: VII SIGRA - Simpósio de Gramados ...Anais. UNESP - Faculdade de Ciências Agronômicas, Botucatu, SP, 2015.

GODOY, L. J. G.; BACKES, C.; ARIGONI, P.; VILLAS BÔAS, R. L.; LIMA, C. P. Doses e modos de aplicação de fertilizante fosfatado na nutrição e produção de tapetes de grama esmeralda. Scientia Agraria Paranaensis, v. 6, n.1-2, p. 77-89, 2007. 
GODOY, L. J. G.; VILLAS BÔAS, R. L. Nutrição de gramados. In: I SIGRA - Simpósio de Gramados ...Anais. UNESP - Faculdade de Ciências Agronômicas, Botucatu, SP, 2003.

GODOY, L. J. G.; VILLAS BÔAS, R. L. Tecnologias para auxiliar o manejo da adubação na produção de gramas. In: V SIGRA - Simpósio de Gramados ...Anais. UNESP - Faculdade de Ciências Agronômicas, Botucatu, SP, 2010.

GURGEL, R. A. G. Principais espécies e variedades de grama. In: I SIGRA - Simpósio de Gramados ...Anais. UNESP - Faculdade de Ciências Agronômicas, Botucatu, SP, 2003.

ILHA SOLTERIA. Novos locais e dias de coleta do lixo reciclável. Disponível em: <http://www.ilhasolteira.sp.gov.br/index.php?option=com_content\&view=article\&id=2 4\&Itemid=20>. Acesso em: 02 de fevereiro de 2018.

ITOGRASS. O tapete natural de grama. 1975. Disponível em: <www.itograss.com. br>. Acesso em: 06 dez. 2016.

JONES, J. B. Turf analysis. Golf course management, v. 48, n. 1, p. 29-32, 1980.

LIMA, C. P. Nutrição, produção e qualidade de tapetes de grama bermuda e esmeralda influenciados pela adubação nitrogenada. 2009. 139f. Tese (Doutorado em Agronomia/Agricultura) - Faculdade de Ciências Agronômicas, Universidade Estadual Paulista, SP, 2009.

LIMA, C. P.; BACKES, C.; VILLAS BÔAS, R. L.; OLIVEIRA, M. R.; KIIHL, T. A. M.; FREITAG, E. E. BACKES, C.; VILLAS BÔAS, R. L.; OLIVEIRA, M. R.; KIIHL, T. A. M.; FREITAG, E. E. Bermuda grass sod production as related to nitrogen rates. Revista Brasileira de Ciência do Solo, Viçosa, v. 34, n. 2, P. 371-378, 2010.

LIMA, C. P.; LOPES, D. A.; VILLAS BÔAS, R. L.; FERNANDES, D. M.; BACKES, C. Medidas de intensidade de coloração verde das folhas, determinadas por dois clorofilômetro em campo de produção de grama esmeralda adubada com doses de ajifer. Tópicos Atuais em Gramados. Ed. FEPAF, Botucatu, p. 170 - 174. 2008.

MACIEL, C. D. G. POletine, J. P.; AQUiNO, C. J. R.; FERREIRA, D. M.; MAIO, R. M. D. Composição florística da comunidade infestante em gramados de Paspalum notatum no município de Assis, SP. Planta daninha, v. 26, n. 1, p. 57-64, 2008. 
MACIEL, C. D. G. Resultados de pesquisa com herbicidas em gramados - um apelo para o registro de produtos. In: GODOY; L. J. G.; MATEUS, C. M. D.; BACKES, C.; VILLAS BÔAS, R. L. Tópicos atuais em gramados II. Ed. FEPAF, Botucatu, p. 115-134, 2010.

MALAVOLTA, E.; VITTI, G. C.; OLIVEIRA, S. A. Avaliação do estado nutricional das plantas: princípios e aplicações. 2. ed. Piracicaba: POTAFOS, 319p., 1997.

MARTELLO, J. M.; CASTILHO, R. M. M. PAGLIARINI, M. K. Pós-colheita de tapetes de grama Esmeralda em relação aos níveis de empilhamento e ambiente de armazenamento. Tecnologia \& Ciência Agropecuária. v.8, n.1, 61-66, 2014.

MILLS, H. A.; JONES, J. B. Plant analysis handbook II: a practical sampling, preparation, analysis and interpretation guide. Athens: MicroMacro, 1996. 456 p.

OLIVEIRA, J. F. V.; CASTILHO, R. M. M.; OLIVEIRA, N. B. Temperatura superficial e do ar em gramado sintético e natural. In: X ENCIVI - Encontro de Ciências da Vida. UNESP - Faculdade de Engenharia de Ilha Solteira, Ilha Solteira, SP, 2016.

PEDRON, F. A. Efeitos da vegetação no ambiente urbano. Agronline.com.br. Disponível em: <http://www.agronline.com.br/artigos/artigo.php?id=88>. Acesso em: 15 de outubro de 2016.

PIEDADE, A. R. Desenvolvimento vegetativo de quatro espécies de gramas irrigadas com efluente de estação de tratamento de esgoto doméstico. 2004. 79p. Dissertação (Mestrado em Agronomia/Irrigação e Drenagem) - Faculdade de Ciências Agronômicas, Universidade Estadual Paulista, Botucatu, 2004.

PIMENTA, C. H. Produção de gramas. In: I SIMPÓSIO SOBRE GRAMADOS - SIGRA, 1. Botucatu, 2003. 7p. (CD-ROM).

QUAGGIO, J.A.; DECHEN, A. R.; RAIJ, B. van. Efeito da aplicação do calcário e gesso sobre a produção de amendoim e lixiviação de bases no solo. Revista Brasileira de Ciência do Solo, v.6, p.189-194, 1982.

RAATS, L. E. S.; PAIVA, P. D. O.; R., M. N. O. Avaliação comparativa de formatos de placas e "plugs" para plantio de grama esmeralda. Revista Brasileira de Horticultura Ornamental, v. 18, n. 2, p. 115-120, 2012. 
RAIJ, B. V. Fertilidade do solo e manejo de nutrientes. 6 ed. Piracicaba-SP: International Plant Nutrition Institute, 2011. 420 p.

REDE CIDADE DIGITAL. Mapa das cidades digitais - São Paulo. Disponível em: $<$ http://redecidadedigital.com.br/mapa_sp.php>. Acesso em: 02 fev. 2018.

SANTOS, L. P. S.; CUBA, R. M. F.; LEITÃO, V. S.; SANTOS NETO, A. S. S. Análise da eficiência de calçadas ecológicas como sistema de drenagem sustentável nos centros urbanos: estudo de caso no Parque Flamboyant, Goiânia-Goiás. Electronic Journal of Management, Education and Environmental Technology (REGET), v. 19, n. 3, p. 837-849, 2015.

SANTOS, P. L. F.; BARCELOS, J. P. Q.; CASTILHO, R. M. M. Diferentes substratos no desenvolvimento de um gramado ornamental para uso em telhados verdes. Periódico Técnico e Científico Cidades Verdes, v. 04, n. 10, p. 81-94, 2016.

SANTOS, P. L. F.; CASTILHO, R. M. M. Caracterização físico-química de diferentes substratos e sua influência no desenvolvimento da grama esmeralda. Tecnologia \& Ciência Agropecuária, João Pessoa, v. 10, n.6, p. 1-5, nov. 2016.

SANTOS, P. L. F.; CASTILHO, R. M. M. Relação entre teor de clorofila e nitrogênio foliar em grama esmeralda cultivada em substratos. Tecnologia \& Ciência Agropecuária, João Pessoa, v. 9, n.2, p. 51-54, set. 2015.

TAIZ, L.; ZEIGER, E. Fisiologia e desenvolvimento vegetal. 6. ed. Porto Alegre: Artmed, 2017. $858 \mathrm{p}$.

UNRUH, J. B. Biologia de gramas de estação quente. In: II SIGRA - Simpósio de Gramados ...Anais. UNESP - Faculdade de Ciências Agronômicas, Botucatu, SP, 2004.

VILLAS BOAS, R. L.; GODOY, L. J. G. Tópicos atuais em gramados. 2. ed. Botucatu: FEPAF, 2008. 203 p.

WATSON, L.; DALLWITZ, M. J. Grass genera of the World: Descriptions. Illustrations, Identification, and Information Retrieval, 1992, 1038 p. 
WINTERS, G. Uso de gramados no paisagismo. In: BACKES, C.; GODOY, L. J. G.; MATEUS, C. M. D.; SANTOS, A. J. M.; VILLAS BÔAS, R. L.; OLIVEIRA, M. R. Tópicos atuais em gramados III: Botucatu/SP: UNESP, Faculdade de Ciências Agronômicas, 2012. p. 201-208.

ZANON, M. E. Desenvolvimento de grama-esmeralda, grama-bermudas'tifway 419' e 'celebration' submetidas a aplicação de reguladores de crescimento. 2015. 58f. Tese (Doutorado em Produção vegetal) - Faculdade de Ciências Agrárias e Veterinárias de Jaboticabal-SP, UNESP, Jaboticabal. 2015.

ZANON, M. E. O mercado de gramas no Brasil, cadeia produtiva, situação e perspectiva. In: I SIGRA - Simpósio de Gramados ...Anais. UNESP - Faculdade de Ciências Agronômicas, Botucatu, SP, 2003. 